\title{
STEP-FORWARD MODALITIES FOR ASSESSMENT OF THE CLINICALLY NODE-NEGATIVE NECK IN HEAD-NECK SQUAMOUS CELL CARCINOMA
}

\author{
SHAH R ${ }^{1}$, FAKIR MAY ${ }^{2}$, KHAN AFM ${ }^{3}$, TALUKDER DC ${ }^{2}$
}

\begin{abstract}
:
"Cancer", till date stands rigidly unconquered despite most strenuous effort and relentless endeavor by the scientists around the world. In global terms head-neck cancer is one of the most common malignancies and represents one of the greatest management challenges for the head and neck surgical oncologists. Most of the head-neck cancers arise in mucosal linings of the oral cavity ( $90 \%$ being squamous cell type) and frequently metastasise to regional lymph nodes in the neck. The presence of nodal metastases is a determinant of prognosis and clinical management. The neck is staged by palpation and imaging, but accuracy of these techniques to detect small metastases is low. In general, 30-40\% of patients will have occult nodal disease and will develop clinically detectable lymph-node metastases when the neck is left untreated. The choice at present is either elective treatment or careful observation followed by treatment of the neck in patients who develop manifest metastases. These unsatisfying therapeutic options have been the subject of debate for decades. Recent developments in staging of the neck, including expression profiling and sentinel lymph-node biopsy, will allow more personalized management of the neck. This review presents "step-forward modalities" or novel diagnostic methods for assessment of the clinically node-negative neck in head-neck squamous cell carcinoma.
\end{abstract}

Key Words: Head-neck cancers, molecular diagnosis and tumour profiling, sentinel lymphnode biopsy

J Dhaka Med Coll. 2012; 21(2) : 218-225.

\section{Introduction:}

Head and neck squamous-cell carcinoma (HNSCC) is a common cancer worldwide. ${ }^{1}$ Risk factors include tobacco smoking, betel nut chewing, excessive alcohol consumption, and human papilloma virus (HPV) infection..$^{2,3}$ Of all HNSCCs, oral squamous-cell carcinoma (SCC) has the highest incidence.

Most tumours in oral-cavity subsites have a comparable high propensity to metastasise to regional lymph nodes in the neck. Findings of recent studies seem to refute the traditional belief that maxillary SCCs have a lower rate of metastasis to the neck. ${ }^{4,5,6}$ The presence of regional neck metastases is a major determinant of both prognosis and treatment decisions in patients with oral SCC. ${ }^{7}$ However, the low sensitivity of currently available diagnostic modalities is a problem, because a fairly high proportion (30-40\%) of lymph-node metastases are left undetected in this population. These metastases will develop into overt neck disease during follow-up.

A policy is in place to treat the neck even when the tumour has been classified clinically as

1. Dr. Rameez Shah, M.S. (Thesis Part), Dept. of ENT \& Head-Neck Surgery, Dhaka Medical College Hospital, Dhaka.

2. Dr. Mohammad Abu Yousuf Fakir, Dr. Debesh Chandra Takukder, Asst. Professor, Dept. of ENT \& Head-Neck Surgery, Dhaka Medical College Hospital, Dhaka.

3. Dr. A. F. Mohiuddin Khan, Professor \& Head, Dept. of ENT \& Head-Neck Surgery, Dhaka Medical College Hospital, Dhaka.

Correspondence: Dr. Rameez Shah, M.S. (Thesis Part) student, Dept. of ENT \& Head-Neck Surgery, Dhaka Medical College Hospital, Dhaka, Email: rameezshah527@yahoo.com 
node-negative (cNO), indicating no disease is detectable in the neck. ${ }^{8}$ This strategy prevents disease in the neck becoming more advanced once previously occult metastases become clinically apparent or are detected late during follow-up. Thus, $60-70 \%$ of patients receive unnecessary treatment, which in the case of neck dissection encompasses a surgical procedure potentially causing disfigurement and associated morbidity. ${ }^{9,10,11}$ The alternative approach of watchful waiting entails careful monitoring of the neck (e.g. by ultrasoundguided fine-needle aspiration cytology during follow-up). ${ }^{12,13}$ Treatment will be given only to patients who develop manifest metastasis, which usually arises within 1-2 years. Although no evidence shows convincingly that one strategy should be preferred over the other, consensus-partly based on an often cited but outdated decision-analysis model-suggests that elective neck treatment is indicated when the chance of occult nodal disease exceeds $20 \% .{ }^{14}$ Hence, increased accuracy to ascertain the metastatic status of the neck will result in fewer unnecessary elective treatments of the neck.

Currently, the neck is staged by palpation and different imaging techniques, including $\mathrm{CT}$, MRI, PET/CT, and ultrasound, which are more accurate than palpation alone. ${ }^{15,16,17}$ Morphological and size criteria are the main determinants of specificity of imaging techniques, whereas sensitivity is limited by the detection threshold. Up to a third of nodal metastases in patients with oral SCC are smaller than the $3 \mathrm{~mm}$ detection threshold that limits sensitivity of available imaging techniques, ${ }^{18}$ and occult neck disease therefore remains a relevant issue. In a metaanalysis of PET/CT for assessment of cervical lymph-node metastasis, ${ }^{16}$ sensitivity of PET/ CT was only $50 \%$ for patients who were nodenegative on palpation, whereas specificity was $87 \%$. The performance of ultrasound, MRI, and $\mathrm{CT}$ was equally disappointing. Later research has shown similar results. ${ }^{19}$

The limitation of imaging techniques to detect small metastatic deposits has led to a search for additional characteristics or biomarkers assessable on the primary tumour to predict nodal disease. Histopathological or molecular features of the primary tumour can predict the presence of nodal metastases in the individual patient, irrespective of the actual size of the tumour. ${ }^{20}$ Measures such as perineural invasion, vascular invasion, tumour border (infiltrative or pushing), tumour thickness, and depth of invasion have been studied extensively for correlations. Only tumour thickness is associated consistently with the presence of nodal metastases. ${ }^{21}$ However, suggested cutoffs for thickness range from 1.5 $\mathrm{mm}$ to $10 \mathrm{~mm}$, and consensus to recommend elective neck dissection is missing. ${ }^{21-24}$ One reason could be that definitions and methods to measure tumour thickness vary in reports. Moreover, tumour thickness is usually ascertained on histopathological examination of the resection specimen, implying a secondstage surgical procedure when elective neck dissection is indicated. Measurement of tumour thickness before surgery, using an intraoral ultrasound probe, could be more promising in this respect. ${ }^{24}$ Besides histological variables, many single molecular markers have been studied to predict the presence of nodal metastasis. In view of the complexity of the metastatic process, no consistent and clinically useful correlations have been noted for any markers. ${ }^{20}$

Thus, the dilemma in current clinical management of the neck is the choice between possible under treatment of $30-40 \%$ of patients with occult metastases and overtreatment of the remaining 60-70\%. Personalised management of the clinically node-negative (cNO) neck, especially in patients with oral SCCs, would benefit greatly from staging techniques that add accuracy to assessment of nodal disease, particularly when these methods are not or are minimally dependent on size of the metastasis. Methods are being developed to diagnose or predict occult metastases in the neck. In this Review Article, we restrict ourselves to discussion of the two most promising techniques, which are arguably ready for clinical implementation and have a very different but complementary nature: (1) gene-expression profiling and (2) sentinel lymph-node biopsy. We describe 
various aspects of these two approaches and suggest a new staging algorithm to incorporate both methods, to optimise management of the cNO neck in patients with early-stage oral SCC.

Molecular diagnosis and tumour profiling:

Gene-expression profiling with DNA microarrays and next-generation sequencing approaches (RNAseq) signal a new era of tumour classification and prognostication. RNA isolated from the tumour specimen can be used to ascertain expression levels of all genes simultaneously in one experiment. This process has led to novel classifications of lymphomas, breast cancer, and HNSCC. ${ }^{25,26,27}$ Furthermore, prognostic profiles have been identified in many tumour types, including breast cancer and HNSCC. ${ }^{28,29}$ Roepman and colleagues ${ }^{30,} 31$ used gene-expression profiling to stage the clinically NO neck in HNSCC patients and, using RNA from the primary tumour specimen, identified particular profiles that could predict N-stage. In a multicentre validation study, undertaken at all head and neck oncological centres in the Netherlands, ${ }^{32}$ the expression profile to predict lymph-node metastasis was transferred to a diagnostic platform to facilitate clinical implementation. Subsequently, the profile was validated with an independent series of 222 samples of oral and oropharyngeal SCC. Although the array platform was changed, the profile predicted N-stage as expected. For the group of cT1-T2NO or early-stage oral SCCs $(n=101)$, a negative predictive value of $89 \%$ was recorded. For these cases the issue of elective neck treatment is most relevant, because early-stage oral SCC is treated by transoral surgery and, thus, there is no need to enter the neck for excision of the primary tumour. Some cancers in the validation series were HPV-positive, and the profile worked less well with HPV-positive tumours. ${ }^{32}$ However, HPV occurs rarely in tumours of the oral cavity, with a prevalence estimated at less than $5 \% .{ }^{3,}{ }^{34}$ Hence, in patients with early-stage (cT1-T2N0) oral SCC, gene-expression profiling might reduce greatly the number of unnecessary elective neck dissections, allowing more personalised treatment.
Improvements can still be made. However, Nstatus was ascertained by routine histopathological examination as reference standard, whereas detection of micrometastases $(0.2-2 \mathrm{~mm})$ can be increased by stepped serial sectioning and immunohistochemistry of all lymph nodes. Such extensive immunohistopathological examination allows detection of 5-58\% (mean $20 \%$ ) of metastases ${ }^{35}$ that escape routine histological detection. Recently, we showed that the predictive power of the profiling approach rises further when histopathological assessment of all lymph nodes is scrutinised (unpublished data). Another improvement of the gene-expression profiling approach might be noted with analysis of multiple biopsy samples. Findings of next-generation sequencing studies indicate that intratumour heterogeneity is present in several cancer types, and this heterogeneity can sometimes be linked to particular areas of the tumour specimen. ${ }^{36-39}$ Intratumour genetic heterogeneity might not necessarily be reflected in global gene-expression profiles, but further studies are needed because the predictive power of gene-expression profiles in early-stage oral SCC could be affected by genetic heterogeneity.

Encouragingly, the highest predictive power for gene-expression profiling was seen in the clinically relevant group of cT1-T2NO oral SCCs. Although this association could be related to the lower prevalence of nodal metastasis in this group, it seems to also make sense from a biological perspective. Early lymphatic dissemination of tumours classified as T1 and T2 might be the direct result of intrinsic tumour properties reflected in specific geneexpression profiles, whereas in advanced cancers, destruction of anatomical barriers and invasive growth is likely to become an important factor associated with lymphatic metastasis as well.

Gene-expression profiling is best undertaken on fresh or frozen tumour samples; arguably, this strategy might restrict applicability, because biopsy samples or surgical specimens are routinely formalin-fixed and paraffinembedded (FFPE). However, taking an additional 
biopsy sample for gene-expression profiling would hardly impose a substantial burden. Moreover, recent developments to extract RNA from FFPE specimens for gene-expression profiling could be helpful. ${ }^{29,40}$

An alternative option would be to ascertain genome-wide genetic changes in DNA, using a comparative genomic hybridisation microarray or next-generation sequencing platform. Switching from RNA to DNA profiling is not an unrealistic idea; the breast cancer classification ascertained by RNA expression profiling has been reproduced accurately by comparative genomic hybridisation microarray DNA profiling, ${ }^{41}$ suggesting that differences in gene-expression profiles between tumours might be reflected well in the number and type of DNA changes. Whether or not this idea also holds true for staging of the cNO neck in patients with oral SCC remains to be established.

Gene-expression profiles of tumour biopsy samples assessed by microarray hybridisation have proven their potential value for $\mathrm{N}$-staging of oral SCC. However, although DNA or protein profiles might be applied likewise, N-stage predictive profiles have not been published, to the best of our knowledge, let alone validated in prospective multicentre studies.

\section{Sentinel lymph-node biopsy:}

In an attempt to more reliably select lymph nodes that potentially contain metastases, sentinel lymph-node assessment, which is used extensively in melanoma and breast cancer, has been introduced. The sentinel lymph node is likely to be the first lymph node to harbour metastasis and can be used to provide information on the rest of the nodal basin. It is usually identified by peritumoral injection of radioactive colloid and blue dye. Preoperative lymphoscintigraphy intraoperative visualisation of blue colouration, and intraoperative radionuclide detection with a gamma probe allow identification of the sentinel lymph node. After surgical removal, this node is studied meticulously by histopathological examination, using stepped serial sectioning and immunohistochemistry. If the sentinel lymph node contains metastatic tumour cells, treatment of the neck is recommended, usually in a second procedure. ${ }^{42}$ The sentinel lymph-node procedure is deemed more precise than imaging procedures and less invasive than elective neck dissection. Moreover, it is associated with significantly less postoperative morbidity and better shoulder function compared with elective neck dissection. ${ }^{43}$

Current best-practice guidelines for provision of sentinel lymph-node biopsy to patients with early-stage oral SCC have been outlined, which provide a framework for the currently evolving recommendations for its use. ${ }^{42}$ To safely avoid elective treatment of the neck in as many patients as possible, a high sensitivity and high negative predictive value are both needed. In the American College of Surgeons Oncology Group Z0360 validation study of 140 patients in 25 institutions, sensitivity was $90 \%$ and the negative predictive value was $96 \%$; these figures were even better for skilled surgeons. ${ }^{44}$ However, in this study, standard histopathological examination of the neckdissection specimen was used as the gold standard. Therefore, occult micrometastasis might have been missed, contributing to higher figures for sensitivity and negative predictive value. In a meta-analysis of 19 pilot studies with a total of 347 oral and oropharyngeal cancer patients, a pooled sensitivity of $92 \cdot 6 \%$ was reported. ${ }^{45}$ After initial studies to validate the sentinel lymph-node approach in patients with early-stage oral SCC, several prospective observational studies have been reported. In two large single-centre studies, sensitivities and negative predictive values of at least $90 \%$ were noted. ${ }^{46,47}$ In these studies, neck dissection was undertaken only when the sentinel lymph node contained metastasis, and a watchful-waiting strategy was followed when the sentinel lymph node was tumour-free.

In a European multicentre study, 33 of 134 patients with cT1/2NO oral SCC, 79 patients underwent sentinel lymph-node biopsy as the sole staging method, whereas 55 underwent sentinel lymph-node biopsy followed by elective neck dissection. For the two groups together, using a reference standard of 5 years follow-up after sentinel lymph-node biopsy staging, a 
sensitivity of $91 \%$ and a negative predictive value of $95 \%$ were recorded. The better performance seen in patients who underwent both techniques (sensitivity 96\%, negative predictive value $97 \%$ ) compared with those who only had sentinel lymph-node biopsy $(87 \%, 94 \%)$ can again be accounted for by use of standard histopathological examination of the neck dissection specimen versus 5 years follow-up as a gold standard for metastasis. Of note, both the sentinel lymph-node identification rate and sensitivity were significantly poorer in patients with floor-of-mouth tumours. Peritumoral injection sites might overshine the sentinel lymph node in these patients, resulting in detection failure by the gamma probes. ${ }^{33}$ The observational multicentre European Sentinel Node Trial (SENT), ${ }^{48}$ with more than 300 patients enrolled, has completed accrual and long-term follow-up data are awaited. Overall, detection rates, sensitivity, and negative predictive values of sentinel lymph-node biopsy procedures are generally above $90 \%$.

Some innovations would improve preoperative imaging of the sentinel lymph node. Hybrid single-photon emission $\mathrm{CT}$ with integrated $\mathrm{CT}$ (SPECT/CT) can augment visualisation of the relation of sentinel lymph nodes to several vital vascular and neural structures, thus enabling safer removal of these nodes. Furthermore, enhanced topographical orientation and delineation of sentinel lymph nodes against surrounding structures might also reduce surgical time.

Although SPECT/CT has the potential to detect more sentinel lymph nodes, it still has some difficulties in visualisation of nodes in close spatial relation to the injection site. ${ }^{49}$ Promising preclinical results have been reported of 89Zr-nanocolloidal albumin-based PET/CT lymphoscintigraphy for sentinel lymphnode detection in HNSCC. ${ }^{50}$

Technical innovations to improve intraoperative localisation of sentinel lymph nodes include intraoperative real-time imaging, freehand SPECT, and fluorescence imaging. Intraoperative real-time imaging with the portable gamma camera provides an overview of all radioactive spots and can show sentinel lymph nodes near the injection site by adjustment of its position. Another advantage might be the certainty it can provide about the completeness and accuracy of sentinel lymphnode excision, by showing the remaining activity. ${ }^{51}$ Freehand SPECT is designed to ascertain the position of the detector relative to the patient, through which threedimensional images are generated. These images provide the surgeon with information about the direction and depth of the sentinel lymph node in relation to the probe. ${ }^{52}$ The feasibility of near-infrared fluorescence-guided sentinel lymph-node detection has been shown in HNSCC, with indocyanine green as fluorescent tracer. ${ }^{53}$ Other tracers with enhanced optical properties have been tested in HNSCC in preclinical settings. ${ }^{50,54}$ The real clinical additional value of these techniques has still to be assessed.

Developments in detection of metastasis in the sentinel lymph-node specimen have been made. While the biopsy procedure is taking place, immediate frozen section, imprint cytology, or molecular techniques can be done that should reliably show sentinel lymph-node metastasis. ${ }^{55}$ Furthermore, a rapid automated quantitative reverse transcriptase PCR assay has been described that took about $35 \mathrm{~min}$ to complete and had an accuracy of $94.2 \%$ for identification of positive and negative nodes, which might be more accurate than intraoperative pathological analysis. ${ }^{56}$ Thus, the surgical procedure-including neck dissection in case of a positive test for nodal metastasis - could be restricted to one session instead of the two currently needed in sentinelnode procedures. However, this change could interfere with current surgical planning efficiency, because complete neck dissection takes about five times longer to undertake than does sentinel lymph-node biopsy of the neck. These technical developments could raise the rate of sentinel lymph-node detection further and facilitate the procedure, potentially resulting in less morbidity for the patient, reduced operating time, and increased convenience for the head and neck surgeon. 


\section{Conclusion:}

Oral SCC is predominantly treated surgically, ${ }^{\mathbf{1 3}}$ therefore, the issue of elective neck dissection versus watchful waiting is most relevant for early-stage (cT1-T2N0) tumours. ${ }^{\mathbf{5 7}, 58}$ In patients with advanced tumours (cT3-T4), who have a fairly high probability of cervical lymph-node metastases and often need neck surgery to access the primary tumour or to reconstruct the surgical defect, most head and neck surgeons will opt for elective neck dissection anyway. ${ }^{\mathbf{5 7}, \mathbf{5 8}}$ Our review does not intend to change this policy. However currently, physical examination and imaging techniques are the most widely accepted and applied methods for assessment of the neck in oral SCC. Limitations in accuracy of these modalities have not led to altered management of the cNO neck in earlystage disease. Combining gene-expression profiling and sentinel lymph-node biopsy in addition to current imaging techniques will further reduce the rate of occult metastasis. In view of the accuracy of these techniques, this rate can be reduced to a level acceptable to allow a wait-and-see policy for the neck in patients with oral SCC classified as T1 and T2. Furthermore, restriction of sentinel lymphnode biopsy to individuals who are classified as node-positive on gene-expression profiling eliminates overtreatment. Prospective clinical trials implementing this staging algorithm are needed to investigate whether gene-expression profiling and sentinel lymph-node biopsy can be combined and whether oncological and functional outcomes in patients with oral SCC will indeed improve.

\section{References:}

1. Jemal A, Bray F, Center MM, Ferlay J, Ward E, Forman D. Global cancer statistics. CA Cancer J Clin 2011; 61: 69-90.

2. Ang KK, Harris J, Wheeler R, et al. Human papillomavirus and survival of patients with oropharyngeal cancer. N Engl J Med 2010; 363: 24-35.

3. Leemans CR, Braakhuis BJ, Brakenhoff RH. The molecular biology of head and neck cancer. Nat Rev Cancer 2011; 11: 9-22.

4. Morris LG, Patel SG, Shah JP, Ganly I. High rates of regional failure in squamous cell carcinoma of the hard palate and maxillary alveolus. Head Neck 2011; 33: 824-30.

5. Montes DM, Carlson ER, Fernandes R, et al. Oral maxillary squamous carcinoma: an indication for neck dissection in the clinically negative neck. Head Neck 2011; 33: 1581-5.

6. Brown JS, Bekiroglu F, Shaw RJ, Woolgar JA, Rogers SN. Management of the neck and regional recurrence in squamous cell carcinoma of the maxillary alveolus and hard palate compared with other sites in the oral cavity. Head Neck 201210.1002/hed.22957. published online Feb 6.

7. Argiris A, Karamouzis MV, Raben D, Ferris RL. Head and neck cancer. Lancet 2008; 371: 1695709 .

8. Ferlito A, Silver CE, Rinaldo A. Elective management of the neck in oral cavity squamous carcinoma: current concepts supported by prospective studies. Br J Oral Maxillofac Surg 2009; 47: 5-9.

9. van Wilgen CP, Dijkstra PU, van der Laan BF, Plukker JT, Roodenburg JL. Morbidity of the neck after head and neck cancer therapy. Head Neck 2004; 26: 785-91.

10. van Wouwe M, de Bree R, Kuik DJ, et al. Shoulder morbidity after non-surgical treatment of the neck. Radiother Oncol2009; 90: 196-201.

11. Bradley PJ, Ferlito A, Silver CE, et al. Neck treatment and shoulder morbidity: still a challenge. Head Neck 2011; 33:1060-7.

12. Nieuwenhuis EJ, Castelijns JA, Pijpers R, et al. Wait-and-see policy for the NO neck in earlystage oral and oropharyngeal squamous cell carcinoma using ultrasonography-guided cytology: is there a role for identification of the sentinel node?. Head Neck 2002; 24: 282-9.

13. Rodrigo JP, Shah JP, Silver CE, et al. Management of the clinically negative neck in early-stage head and neck cancers after transoral resection. Head Neck 2011;33: 1210-9.

14. Weiss MH, Harrison LB, Isaacs RS. Use of decision analysis in planning a management strategy for the stage NO neck. Arch Otolaryngol Head Neck Surg 1994; 120: 699-702.

15. de Bondt RB, Nelemans PJ, Hofman PA, et al. Detection of lymph node metastases in head and neck cancer: a meta-analysis comparing US, USgFNAC, CT and MR imaging. Eur J Radiol 2007; 64: 266-72.

16. Kyzas PA, Evangelou E, DenaxaKyza D, Ioannidis JP. 18F-fluorodeoxyglucose 
positron emission tomography to evaluate cervical node metastases in patients with head and neck squamous cell carcinoma: a meta-analysis. J Natl Cancer Inst 2008;100: 712-20.

17. Ng SH, Yen TC, Chang JT, et al. Prospective study of [18F] fluorodeoxyglucose positron emission tomography and computed tomography and magnetic resonance imaging in oral cavity squamous cell carcinoma with palpably negative neck.J Clin Oncol 2006; 24: 4371-6.

18. Buckley JG, MacLennan K. Cervical node metastases in laryngeal and hypopharyngeal cancer: a prospective analysis of prevalence and distribution. Head Neck 2000; 22: 380-5.

19. Liao LJ, Lo WC, Hsu WL, Wang CT, Lai MS. Detection of cervical lymph node metastasis in head and neck cancer patients with clinically NO neck-a meta-analysis comparing different imaging modalities. BMC Cancer 2012; 12: 236.

20. Takes RP, Rinaldo A, Rodrigo JP, Devaney KO, Fagan JJ, Ferlito A. Can biomarkers play a role in the decision about treatment of the clinically negative neck in patients with head and neck cancer?. Head Neck 2008; 30: 525-38.

21. Melchers LJ, Schuuring E, van Dijk BA, et al. Tumour infiltration depth $>/=4 \mathrm{~mm}$ is an indication for an elective neck dissection in pT1cNO oral squamous cell carcinoma. Oral Oncol 2012; 48: 337-42.

22. Pentenero M, Gandolfo S, Carrozzo M. Importance of tumor thickness and depth of invasion in nodal involvement and prognosis of oral squamous cell carcinoma: a review of the literature. Head Neck 2005; 27: 1080-91.

23. Huang SH, Hwang D, Lockwood G, Goldstein DP, O'Sullivan B. Predictive value of tumor thickness for cervical lymph-node involvement in squamous cell carcinoma of the oral cavity: a meta-analysis of reported studies. Cancer 2009; 115: 1489-97.

24. Lodder WL, Teertstra HJ, Tan IB, et al. Tumour thickness in oral cancer using an intra-oral ultrasound probe. Eur Radiol2011; 21: 98-106.

25. Alizadeh AA, Eisen MB, Davis RE, et al. Distinct types of diffuse large B-cell lymphoma identified by gene expression profiling. Nature 2000; 403: 503-11.

26. Perou CM, Sorlie T, Eisen MB, et al. Molecular portraits of human breast tumours. Nature 2000; 406: 747-52.

27. Chung $\mathrm{CH}$, Parker JS, Karaca G, et al. Molecular classification of head and neck squamous cell carcinomas using patterns of gene expression. Cancer Cell 2004; 5: 489-500.
28. van't Veer LJ, Dai H, van de Vijver MJ, et al. Gene expression profiling predicts clinical outcome of breast cancer. Nature2002; 415: 530-6.

29. Chung $\mathrm{CH}$, Parker JS, Ely K, et al. Gene expression profiles identify epithelial-tomesenchymal transition and activation of nuclear factor-êB signaling as characteristics of a highrisk head and neck squamous cell carcinoma. Cancer Res 2006; 66: 8210-8.

30. Roepman P, Wessels LF, Kettelarij N, et al. An expression profile for diagnosis of lymph node metastases from primary head and neck squamous cell carcinomas. Nat Genet 2005; 37: 182-6.

31. Roepman P, Kemmeren P, Wessels LF, Slootweg PJ, Holstege FC. Multiple robust signatures for detecting lymph node metastasis in head and neck cancer. Cancer Res 2006; 66: 2361-6.

32. van Hooff SR, Leusink FK, Roepman P, et al. Validation of a gene expression signature for assessment of lymph node metastasis in oral squamous cell carcinoma. J Clin Oncol 2012 10.1200/JCO.2011.40.4509. published online Oct 8 .

33. Alkureishi LW, Ross GL, Shoaib T, et al. Sentinel node biopsy in head and neck squamous cell cancer: 5-year follow-up of a European multicenter trial. Ann Surg Oncol 2010; 17: 2459-64.

34. Herrero R, Castellsague X, Pawlita M, et al. Human papillomavirus and oral cancer: the International Agency for Research on Cancer multicenter study. J Natl Cancer Inst 2003; 95: 1772-83.

35. Ferlito A, Rinaldo A, Devaney KO, N akashiro K, Hamakawa H. Detection of lymph node micrometastases in patients with squamous carcinoma of the head and neck. Eur Arch Otorhinolaryngol 2008; 265: 1147-53

36. Yachida S, Jones S, Bozic I, et al. Distant metastasis occurs late during the genetic evolution of pancreatic cancer. Nature2010; 467: 1114-7.

37. Gerlinger M, Rowan AJ, Horswell S, et al. Intratumor heterogeneity and branched evolution revealed by multiregion sequencing. $\mathrm{N}$ Engl J Med 2012; 366: 883-92.

38. Nik-Zainal S, Alexandrov LB, Wedge DC, et al. Mutational processes molding the genomes of 21 breast cancers. Cell 2012;149: 979-93.

39. Nik-Zainal S, Van Loo P, Wedge DC, et al. The life history of 21 breast cancers. Cell 2012; 149: 994-1007.

40. Xie Y, Xiao G, Coombes KR, et al. Robust gene expression signature from formalin-fixed paraffinembedded samples predicts prognosis of nonsmall-cell lung cancer patients. Clin Cancer Res 2011; 17: 5705-14. 
41. Smeets SJ, Harjes U, van Wieringen WN, et al. To DNA or not to DNA? That is the question, when it comes to molecular subtyping for the clinic!. Clin Cancer Res 2011; 17: 4959-64.

42. Alkureishi LW, Burak Z, Alvarez JA, et al. Joint practice guidelines for radionuclide lymphoscintigraphy for sentinel node localization in oral/oropharyngeal squamous cell carcinoma. Ann Surg Oncol 2009; 16: 3190-210.

43. Murer K, Huber GF, Haile SR, Stoeckli SJ. Comparison of morbidity between sentinel node biopsy and elective neck dissection for treatment of the n0 neck in patients with oral squamous cell carcinoma. Head Neck 2011; 33: 1260-4.

44. Civantos FJ, Zitsch RP, Schuller DE, et al. Sentinel lymph node biopsy accurately stages the regional lymph nodes for T1-T2 oral squamous cell carcinomas: results of a prospective multiinstitutional trial. J Clin Oncol 2010; 28: 13951400 .

45. Paleri V, Rees G, Arullendran P, Shoaib T, Krishman S. Sentinel node biopsy in squamous cell cancer of the oral cavity and oral pharynx: a diagnostic meta-analysis. Head Neck 2005; 27: 739-47.

46. Kovacs AF, Stefenelli U, Seitz O, et al. Positive sentinel lymph nodes are a negative prognostic factor for survival in T1-2 oral/oropharyngeal cancer-a long-term study on 103 patients. Ann Surg Oncol 2009; 16: 233-9.

47. Broglie MA, Haile SR, Stoeckli SJ. Long-term experience in sentinel node biopsy for early oral and oropharyngeal squamous cell carcinoma. Ann Surg Oncol 2011; 18: 2732-8.

48. Gurney BAS, Schilling C, Putcha V, et al. Implications of a positive sentinel node in oral squamous cell carcinoma. Head Neck 2012 10.1002/hed.21973. published online Jan 31.

49. Haerle SK, Hany TF, Strobel K, Sidler D, Stoeckli SJ. Is there an additional value of SPECT/CT over planar lymphoscintigraphy for sentinel node mapping in oral/oropharyngeal squamous cell carcinoma?. Ann Surg Oncol 2009; 16: 3118-24.
50. Heuveling DA, Visser GW, Baclayon M, et al. ${ }^{89} \mathrm{Zr}-$ nanocolloidal albumin-based PET/CT lymphoscintigraphy for sentinel node detection in head and neck cancer: preclinical results. J Nucl Med 2011; 52: 1580-4.

51. Vermeeren L, Valdes Olmos RA, Klop WM, Balm AJ, van den Brekel MW. A portable gammacamera for intraoperative detection of sentinel nodes in the head and neck region. J Nucl Med 2010; 51: 700-3.

52. Heuveling DA, Karagozoglu KH, van Schie A, van Weert S, van Lingen A, de Bree R. Sentinel node biopsy using 3D lymphatic mapping by freehand SPECT in early stage oral cancer: a new technique. Clin Otolaryngol 2012; 37: 89-90.

53. van den Berg NS, Brouwer OR, Klop WM, et al. Concomitant radio- and fluorescence-guided sentinel lymph node biopsy in squamous cell carcinoma of the oral cavity using ICG- $(99 \mathrm{~m}) \mathrm{Tc}-$ nanocolloid. Eur J Nucl Med Mol Imaging 2012; 39: 1128-36.

54. Keereweer S, Hutteman M, Kerrebijn JD, van de Velde CJ, Vahrmeijer AL, Lowik CW. Translational optical imaging in diagnosis and treatment of cancer. Curr Pharm Biotechnol 2012; 13: 498-503.

55. Vorburger MS, Broglie MA, Soltermann A, et al. Validity of frozen section in sentinel lymph node biopsy for the staging in oral and oropharyngeal squamous cell carcinoma. J Surg Oncol 201210.1002/jso.23156. published online May 14.

56. Ferris RL, Xi L, Seethala RR, et al. Intraoperative qRT-PCR for detection of lymph node metastasis in head and neck cancer. Clin Cancer Res 2011; 17: 1858-66.

57. Bar Ad V, Chalian A. Management of clinically negative neck for the patients with head and neck squamous cell carcinomas in the modern era. Oral Oncol 2008; 44: 817-22.

58. Genden EM, Ferlito A, Silver CE, et al. Contemporary management of cancer of the oral cavity. Eur Arch Otorhinolaryngol 2010; 267: 1001-17. 